Bangladesh Journal of Neuroscience 2011; Vol. 27 (2) : 74-77

\title{
Prevalence of Analgesic Induced Deafness
}

\author{
SEHELLY JAHAN ${ }^{1}$, MUHAMMAD FARIDUDDIN MILKY ${ }^{2}$
}

\begin{abstract}
:
Background: Analgesic drugs may produce toxic effect on the cochlear system depending on the dose, duration or concomitant renal failure. Objective: Our aim was to find out the relationship between analgesic use and hearing loss. Methods: This was a cross sectional study done combindly between E.N.T. and Neurology department of Bangladesh Medical college among the patients complaining of hearing loss of different degree. One hundred and forty four patients of both sexes having sensory-neural type of hearing loss were included in this study. Results: Out of 944 patients, eighty eight patients were taking tab. Diclofenac (61.11\%), thirty six patients were taking Naproxen(25\%), and twelve were taking Paracetamol (8.33\%) eight were taking Ibuprophen(5.55\%). Eighty eight patients were taking the drugs more than two years (61\%). All patients were taking analgesic 3-4 times per week. Intensity of damage was severe in 52.77\%; moderate damage was evident in $30.55 \%$ cases and mild damage found in $16.66 \%$ cases. Severity of damage was moderate to severe in most cases of Diclofenac. Conclusion: Analgesic drugs may have toxic effect on hearing.
\end{abstract}

Key Words: Analgesic, deafness.

Introduction:

Hearing is one of the most precious possessions of man. So prevention of deafness should be the primary target of an Otologist as well as others. Deafness refers to conditions in which individuals are fully or partially unable to detect or perceive at least some frequencies of sound which can typically be heard by members of their species. Conductive deafness indicates impaired hearing due to dysfunction of conduction of sound waves through outer ear, eardrum or bones of the middle ear .Sensory-neural deafness results from diseases of the inner ear specially the cochlea where the sound vibrates and converted into neural signals or any part of the brain which subsequently process the signals ${ }^{1}$.

Organ of corti is the end organ of hearing wherein sound is transduced into nerve impulse. It consists of neuro-epithelial cells, called hair cells that rest on the basilar membrane. Sound causes basilar membrane to vibrate and stimulate the hair cells. This stimulation is then transmitted to the cochlear nerve. Basillar membrane vibrates at different frequencies according to the sound stimulus, so the cochlear nerve can differentiate and resolve complexes of sounds ${ }^{2}$.
Sensory neural deafness also called neural deafness which is due to the disease of the cochlea or cochlear division of the vestibule-cochlear nerve or brain. Early Sensory-neural deafness is characterized by partial loss of perception of high pitched sounds which can be ascertained by tuning forks of different frequencies but most accurately by the use of audiometer and construction of an audiogram which reveals the entire range of hearing at a glance.

Everyday most persons are taking various types of analgesics for different reasons. Most of them along with analgesia cause significant damage to many organs of the body including hearing. Factors affecting ototoxicity include dose, duration of therapy, concurrent renal failure, infusion rate, and life time dose, co-administration of drugs having ototoxic potential and genetic susceptibility. Elderly and people with pre-existing hearing loss, ototoxic drugs should be used if no alternative is available. Lowest effective dose should be used and levels should be closely monitored ${ }^{3}$.

Over 130 drugs and chemicals are reported to be potentially ototoxic and many cause permanent and temporary structural damage of the inner ear. Damage can be of various degree and reversibility ${ }^{4}$.

1. Associate Professor of Neurology, Bangladesh Medical College \& Hospital

2. Consultant ENT, Bangladesh Medical College Hospital 


\section{Materials and Method:}

It was a prospective study done among the patients coming for treatment of hearing deficit in Bangladesh Medical College. Neurology and E.N.T. department combindly conducted the study during period of July 2008 to June 2009. Proper history was taken through a structured questionnaire including their job specification and the primary disease for which they were taking the analgesics. Elaborate drug history was taken including their dose, duration and frequency of use .History of concomitant nephropathy was noted. Audiometry was done in each patient and degree of hearing loss was noted.

\section{Inclusion Criteria}

1. Patient complaining of deafness was included.

2. Patient taking analgesic for at least twice weekly for six months was included.

\section{Exclusion Criteria:}

1. Patient having family $\mathrm{H} / \mathrm{O}$ deafness was excluded.

2. Patient more than 70 yrs was excluded.

3. Deafness following Meningitis, Encephalitis, and Stroke were excluded.

4. Known cases of C.S.O.M. or ruptured tympanic membrane were excluded.

\section{Results:}

A total of one hundred and forty four patients were included in this study. Among them seventy six patients were more than fifty years of age $(52.77 \%)$ and seventy two patients were male (50\%). Forty of them were housewives suffering from headache and backache $(27.77 \%)$. Thirty two of them were serving in different marketing departments, doing frequent journey mostly by motor cycle and suffering from headache, body ache and backache (22.22\%). Seventy two of them were sedentary worker (50\%) (Table -I). Thirty two of them were suffering from migraine (22.22\%), forty had backache $(27.77 \%)$, thirty two had diabetic neuropathy (22.22\%) and another forty had osteoarthritis, myalgia (28.77\%) (Table -II). Eighty eight patients were taking tab. Diclofenac(61.11\%), thirty six patients were taking Naproxen(25\%), and twelve were taking Paracetamol (8.33\%) eight were taking Ibuprophen(5.55\%) .Eighty eight patients were taking the drugs more than two years ((61\%). All patients were taking analgesic 3-4 times per week. Intensity of damage was severe in $52.77 \%$; moderate damage was evident in $30.55 \%$ cases and mild damage found in $16.66 \%$ cases. . Concomitant nephropathy was present in twenty patients (table-III).

Table-I

Demographic characteristics of Patients ( $n=144)$

\begin{tabular}{llcc}
\hline Age &.$>50$ & 76 & $52.77 \%$ \\
& $<50$ & 68 & $475.23 \%$ \\
Sex & male & 72 & $50 \%$ \\
& female & 72 & $50 \%$ \\
\multirow{2}{*}{ Occupation } & Housewife & 40 & $27.77 \%$ \\
& Marketing officer & 32 & $22.22 \%$ \\
& Sedentary job & 72 & $50.00 \%$ \\
\hline
\end{tabular}

Table-II

Primary diagnosis of affected patients $(n=144)$

\begin{tabular}{lll}
\hline Migraine & 32 & $22.22 \%$ \\
.Low back pain & 40 & $27.77 \%$ \\
Diabetic neuropathy & 32 & $22.22 \%$ \\
Osteoarthritis / Myalgia & 40 & $28.77 \%$ \\
\hline
\end{tabular}

Table-III

Details of analgesics used $(n=144)$

\begin{tabular}{llcc}
\hline Name of & Diclofenac & 88 & $61.11 \%$ \\
analgesic & Naproxen & 36 & $25.00 \%$ \\
& Paracetamol & 12 & $8.33 \%$ \\
& Ibuprofen & 8 & $5.55 \%$ \\
Duration of & $>2$ yrs & 88 & $61.11 \%$ \\
use & $<2$ yrs & 56 & $38.88 \%$ \\
Severity of & Mild & 24 & $16.66 \%$ \\
Hearing loss & Moderate & 44 & $30.55 \%$ \\
& Severe & 76 & $52.77 \%$ \\
\hline
\end{tabular}

\section{Discussion:}

There is strong relationship between age and reported hearing loss. $18 \%$ of American adults between $45-64$ yrs, 30\% between $65-74$ and $47 \%$ are more than $75 \mathrm{yrs}$ of age having hearing impairment ${ }^{4}$. In 2005 about 278 million people had 
moderate to profound hearing impairment, $80 \%$ of them used to live in low and middle income countries. The poor suffer more from hearing impairment, because they can't afford preventive and routine care to avoid hearing loss nor the hearing aids to manage the disability ${ }^{5}$. Patients with diagnosed hearing loss in 26,917 men aged 40-74 yrs at baseline in 1986 having regular use of aspirin, NSAID and acetaminophen. regular use of each analgesic was independently associated with increased hearing loss. Multivariate adjusted hazard ratio of hearing loss in regular users ( $2+$ times/wk) compared with men $<2$ times per wk, where 1.12 (95\% confidence interval, 1.04-1.20 for aspirin, 1.21(95\% confidence interval (1.11-1.33) for NSAID and $1.22(95 \%$ confidence interval 1.07-1.39 for acetaminophen. For NSAID and acetaminophen risk increased with longer duration and regular use and magnitude is substantially higher in younger men less than $50 \mathrm{yrs}$ of age ,the hazard ratio of hearing loss was 1.33 for aspirin, 1.61 for NSAID and 1.99 for acetaminophen ${ }^{6}$.

Study showed the average spectrum of electrophysiological cochleo-neural activity (ASECA) decreased during hrs after salicylet administration. At the end of treatment acoustic tuning of ASECA showed partially decreased sensitivity. Risk of hearing loss was assessed in 26,000 using aspirin, acetaminophen and ibuprofen and followed up every two years for eighteen yrs. Results revealed $33 \%$ more hearing loss in regular users $<50$ and between 50-59 but no association with $>60$ and older people .For NSAID regular users aged under 50 were $61 \%$ and $50-5932 \%$ and 60 and older $16 \%$ in regular users than non regular users. For acetaminophen regular users $<50$ 99\% $50-5938$ $\%$ and 60 and $>6016 \%$. 6 The hearing loss is slight to moderate, bilaterally symmetrical, affect all frequencies with affection to high frequencies ${ }^{7}$.

An n/o NSAID have been reported as inducer of aseptic meningitis specially lbuprofen and ketorolac. A $70 \mathrm{yr}$ old male presented with acute meningitis 5 times in 6 months with polymorpho-nuclear leucocytosis and raised protein without any evidence of infection or other causes of meningitis ${ }^{8}$. In another case report of a 66yrs old female for sudden bilateral sensory-neural deafness, she was taking Ibuprofen for three months. M.R.I. of brain revealed abnormal enhancement of the dura and basal cisterns. C.S.F. revealed evidence of asceptic meningitis. Audiogram showed new bilateral sensory-neural deafness. Hearing loss and tinnitus resolved and no abnormality was present in M.R.I. when NSAID discontinued ${ }^{9}$. In another case report recurrent aseptic meningitis temporarily occurred with ibuprofen in a $51 \mathrm{yr}$ old white man presented with acute confusion and aphasia. C.S.F. revealed lymphocytic pleocytosis and raised protein level ${ }^{10}$. Side effect like aseptic meningitis is less in case of diclofenac than other NSAIDS. A survey was done in 7.6 million people world-wide by CIBA-GEIGY ${ }^{11}$.

Aspirin and other NSAID including diclofenac is associated with tinnitus and hearing loss. In two double blind multi central study, diclofenac compared with aspirin and naproxen in patients with $\mathrm{Rh}$. Arthritis. Significantly fewer patients $(p<$ or equal to 0.05 ) discontinued the trial due to tinnitus and deafness in case of diclofenac compared to aspirin and naproxen ${ }^{12}$. A $29 \mathrm{yr}$ male were reported to have sensory-neural deafness after using diclofenac for his post operative pain ${ }^{13}$. In one case report an otherwise healthy patient experienced permanent sensory neural hearing loss after a brief period of naproxen ${ }^{14}$. In another study five patients suffered from hearing loss receiving naproxen, only two recovered after discontinuation of drug ${ }^{15}$. Another double blind comparative study between salicylate and diclofenac for 8 weeks in 301 patients, 9 patients discontinued diclofenac due to tinnitus and hearing loss $^{16}$.

\section{Conclusion:}

Pain is very tough to tolerate specially by busy persons, so they try to get rid of it by either way. But most of them have various side effects including hearing loss. Some study showed hearing improvement after stopping the medicine and few cases not. In many cases it is unpredictable. So there should be a big campaign regarding acknowledgement of the people not to use medicine specially over the counter medicine which is not followed up by any doctor search of toxicity. 


\section{References:}

1. Alan HR, Robert HB. Deafness and Dizziness and disorders of equilibrium. Adams and Victors principles of Neurology $.9^{\text {th }} \mathrm{ed}$. Mc Graw Hill .15:246-8

2 John S. Oghalai, Drug induced oto-toxicity and prevention, Merck Manuals online medical library, July 2007

3. García, V. P., Martínez, F.A., Agusti, E. B., Mencía, L.A. \& Asenjo, V.P. Drug-induced ototoxicity: Current status. Acta Otolaryngologica 2001;121, 569-2

4. Battey JF. National institute on deafness and other communication disorders, Advisory Council, National Institute of Health, Bethosde MD, January 2008.

5. WHO media centre. E- mail: Mediaenquires @WHO.net

6. Curhan SG, Eavey R, Sharorodsty $\mathrm{J}$ et al. Analgesic use and risk of hearing loss in men . The American journal of medicine 2010;123(3) 231-7

7. Cazals $Y$, Auditory sensorineural alterations induced by salicylet ,Prog Neurobiol 2000 Dec; 62 (6):583-1

8. Vargas-machuca E, Mondejar Marin B, Navarro-munoz et al. A Recurrent aseptic meningitis secondary to taking Ibupruphen and ketorolac. Rev Neurol. 2006 Feb; 1628;42(4):217-9
9. Davison SP, Marion M. Sensoryneural hearing loss caused by hearing loss caused by NSAID induced aseptic meningitis. Ear nose and throat journal 1998 Oct; 77(10): 824-6

10. Nguyen HT,Juurlink DN , Recurrent Ibuprofen induced aseptic Meningitis. Ann Pharmacother 2004; 38(3): 408-10

11. William M, Obrien MD . Adverse reactions and NSAID drugs, Diclofenac compared with other NSAID drugs. American journal of medicine 1986;80(4):70-80

12. Kolodny AL, Two double blind trials of Diclofenac sodium with Aspirin and with Naproxen in the treatment of patients with rheumatoid arthritis. J. Rheumatol 1988 Aug; 15(8): 1205-11

13. Pau H., Selvadurai $D$, Reversible sensory nuronal heaving loss following non-otological surgery. Murty LF. Postgrad Med J. 2000; 76: $304-6$

14. Mckinnon BJ and Lassen LF. Naproxen associated sudden sensoryneural hearing loss, Mil Med 1998; 163: 792-3

15. Chapman P. Naproxen and sudden hearing loss. J Laryngol Otol 1982,96: 163-6

16. Bombardier C, Peloso PM, Goldsmith $\mathrm{CH}$. Salasate, a nonacetylated salieylate is as efficatious as diclofenac in patients with rheumatoid arthrities. Salasate-Diclofenac study group. J. Rheumatol 1995; 22(4) :617-24. 